\title{
CATÁLOGO DEL MACROFITOBENTOS DEL MEDITERRÁNEO DE MARRUECOS
}

\author{
Juan Antonio GONZÁLEZ GARCÍA y Francisco CONDE POYALES
}

\begin{abstract}
RESUMEN. Catálogo del macrofitobentos del Mediterráneo de Marruecos. Se ha realizado un estudio del macrofitobentos en el litoral mediterráneo de Marruecos. Como resultado del mismo se han catalogado 335 taxa, de los que 140 son novedades corológicas para la zona. De ellos, 203 corresponden a las Rhodophyceae, 62 a las Phaeophyceae, 66 a las Chlorophyceae y 4 a las Liliopsida. El R/F de 3,27 da un valor muy similar al 3,29 del litoral mediterráneo español.
\end{abstract}

Palabras clave. Macrofitobentos marino, Mar Mediterráneo, Marruecos, algas, fanerógamas marinas.

ABSTRACT. Macrophytobenthic check-list of the Mediterranean coast from Morocco. Macrobenthic marine taxa from Mediterranean Morocco coast are compiled. Number of taxa is 335 (203 Rhodophyceae, 62 Phaeophyceae, 66 Chlorophyceae and 4 Liliopsida). New algae records are 140 (99, 19 and 22 respectively). The R/P ratio is 3.27 , close to the value founded in the Spanish coast Mediterranean Sea (3.29).

Key words. Macrobenthic marine, Mediterranean Sea, Morocco, algae, seagrass.

RÉSUMÉ. Liste du macrophytobenthos du Méditerranée au Maroc. Le nombre de taxa étudiés du macrophytobenthos marine du Méditerranée maroccaine a été de 335 (203 Rhodophyceae, 62 Phaeophyceae, 66 Chlorophyceae et 4 Liliopsida). Les algues nouvelles pour la flore du litoral méditerranéenne maroccaine sont 140 (99, 19 et 22 respectivement). Le R/P est 3.27, un valeur très prochain au Méditerranée de l'Espagne (3.29). De même a une grande ressemblance avec la flore marine du littoral d'Almería (Espagne).

Mots clés. Macrophytobenthos marine, Mer Méditerranée, Maroc, algues, phanérogames marines.

\section{INTRODUCCIÓN}

Son escasos los trabajos que sobre flora bentónica se han realizado en el litoral mediterráneo de Marruecos, por lo que son pocas las especies de algas citadas en dicha área, correspondiendo la mayoría de ellas a localidades muy concretas y reiterativas y a grupos taxonómicos determinados.

Entre dichos trabajos podemos destacar los de Bornet (1892) sobre algunas especies recolectadas en las proximidades de Ceuta; Debray (1897) sobre algas de Tánger y Ceuta; Hariot $(1909,1911)$ sobre las costas de Marruecos en general; De Buen (1913) y Sauvageau (1913, 1920) sobre Fucales y Laminariales del mediterráneo marroquí; Lemoine (1926) y Hamel \& Lemoine (1952) sobre Coralináceas; Bellón (1925) sobre Laminariales; Lozano Cabo (1953) sobre la 
Mar Chica de Melilla-Nador; Werner (1962) sobre Ceuta y Melilla; Conde (1984) sobre las Islas Chafarinas; Navarro \& Gallardo (1989) sobre Tetuán y las Islas Chafarinas; González García y Conde (1991) estudian el macrofitobentos de la Mar Chica de MelillaNador y González García y Conde(1992 y 1993) sobre Fucales y Laminariales.

\section{MATERIAL Y MÉTODOS}

El muestreo de la zona estudiada se ha llevado a cabo durante 5 años (1989-93), con recolecciones a lo largo de todo el litoral (fig. 1 y anexo 1) y durante todos los meses. La mayoría del material fue recolectado in situ con ayuda de escafandra autónoma y sólo en algunos casos se estudió material arrojado o de arrastre. Los puntos 30, 31, 32 y 33 son exclusivamente bibliográficos.

Para la nomenclatura general hemos seguido básicamente a Perret-Boudouresque \& Seridi (1989), con las modificaciones aportadas para Chlorophyceae por Burrows (1991) y Gallardo et al.(1993); para Fucophyceae por Ribera et al. (1992); para Acrochaetiales por Conde (1991); para Corallinales por Silva \&
Johansen (1986) y para Ceramiales por Maggs \& Homersand (1993).

Para cada especie incluimos datos sobre a) corología (fig. 1 y anexo1), b) autoecología según Boudouresque 1984 (anexo 2), c) epifitismo,d) datos fitogeográficos (anexo 3) y e) algunos comentarios particulares.

Todo el material estudiado se encuentra depositado en el Herbario del Departamento de Biología Vegetal de la Universidad de Málaga (Phyc MGC). Las especies con asterisco $\left(^{*}\right)$ representan novedades corológicas para la zona estudiada.

Anexo 1: Relación de puntos de muestreos citados en la corología de cada especie: 1.- Saidia. 2.Cabo de Agua. 3.- Karia Arkemanne (Peña del Burro). 4.- Mar Chica (Altos del Galán). 5.- Mar Chica (Atalayón). 6.- Mar Chica (Nador). 7.- Mar Chica (Sustrato móvil). 8.- Puerto de Melilla. 9.Punta de Rostrogordo.10.- Muelle Colorado. 11.Punta de Sabinilla. 12.- Cala Blanca. 13.- Punta Bermeja. 14.- Punta de la Mina. 15.- Cala Viñas. 16.- Los Farallones. 17.- Cabo de Tres Forcas. 18.Cala Charranes. 19.- Cazaza. 20.- Punta Negri. 21.- Sammar. 22.- Punta de Afrau. 23.- Cabo Quilates. 24.- Cala Bonita. 25.- Playa del Quemado. 26.- Puerto de Alhucemas. 27.- Playa de la Cebadilla. 28.- Cala Iris. 29.- Punta de Pescadores. 30, 31 y 32.- Tetuán. 33.- Ceuta. 34.-Islas Chafarinas. MD.- por casi todo el litoral estudiado.

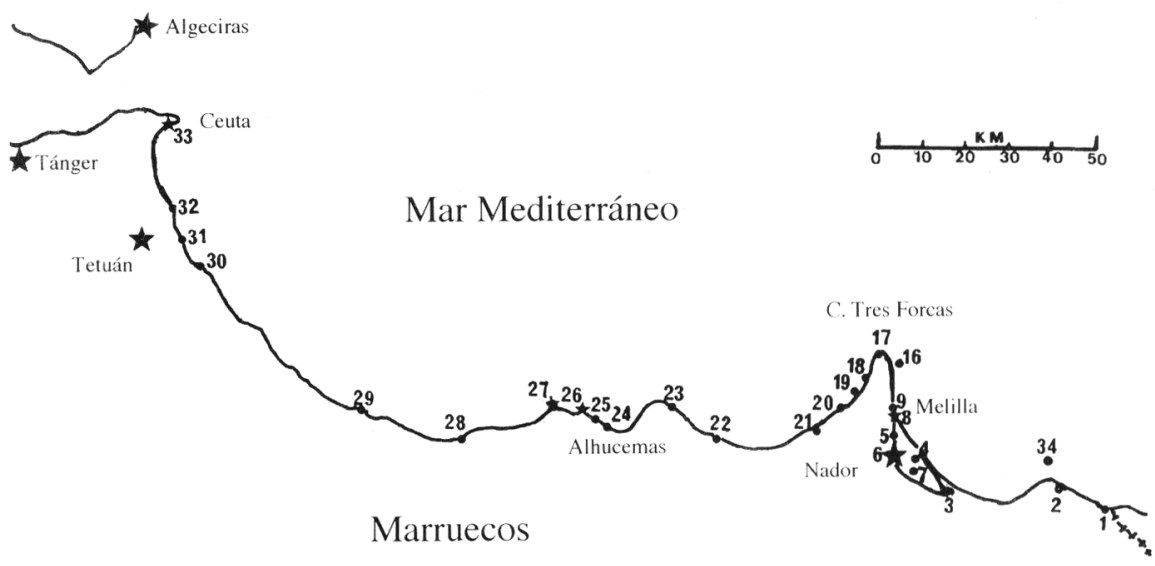

Figura 1. Costas mediterráneas de Marruecos. Situación de las localidades muestreadas. Morocco mediterranean coasts. Study site location. 
Anexo 2: Autoecología según la terminología de Boudouresque (1984): AS: Antiesciófilos; CC: Concreciones coralígenas; CCT: Concreciones coralígenas tolerantes; EM: Cornisa mediolitoral; ETN: Eutrofo y tionitrófilo; FM: Franja mediolitoral; HP: Comunidades de hojas de Posidonia; HSPP: Hemiesciófilo de pequeños puertos; ISR: Infralitoral de sustratos duros; PhI: Fotófilo infralitoral; PhIB: Fotófilo infralitoral batido; PhIC: Fotófilo infralitoral relativamente calmo; PhIG: Fotófilo infralitoral de céspedes; PhIM: Fotófilo infralitoral termófilo de sustratos blandos; PhIP: Fotófilo infralitoral portuario; PhIT: Fotófilo infralitoral termófilo; RM: Roca mediolitoral; RMM1: Roca mediolitoral media 1; RMM2: Roca mediolitoral media 2; RMMI: Roca mediolitoral inferior; SC: Esciófilo de medios calmos; SCI: Esciófilo infralitoral de medios calmos; SCIT: Esciófilo infralitoral de medios calmos y tolerantes; SI: Esciófilo infralitoral; SIC: Esciófilo infralitoral y circalitoral; SM: Esciófilo de sustratos blandos; SRh: Esciófilo reófilo; SSB: Esciófilo superficial batido; SSBc: Esciófilo superficial batido de afinidades cálidas; SSBf: Esciófilo superficial batido de afinidades frías.

Anexo 3: Elementos fitogeográficos según Gayral(1966), Ardré(1969-70) y Giaccone et al., (1985). A: Atlántico; Ab: Atlántico boreal; Abt: Atlántico boreo-tropical; Ao: Holo-Atlántico; APo: Holo-Atlántico-Pacífico; APt: Atlántico-Pacífico tropical; APtf: Atlántico-Pacífico templado frío; At: Atlántico tropical; Atf: Atlántico templadofrío; C: Cosmopolita; CB: Circumboreal; CBA: Circumboreo-austral; IAo: Holo Indo Atlántico; IAt: Indo-Atlántico tropical; IAtf: Indo-Atlántico de afinidades templado frías; IP: Indo-Pacífico; M: Mediterráneo; P: Pantropical; SC: Subcosmopolita.

\section{CATÁLOGO FLORÍSTICO}

\section{RHODOPHYCEAE}

\section{ACROCHAETIALES}

\section{* Audouinella bonnemaisoniae (Batters) Dixon} = Acrochaetium bonnemaisoniae (Batters) J. Feldm. et G. Feldm.

a) 12. b) PhI. c) Epífita de Pterocladia capillacea. d) Ab. e) Citada como A. endophytica por Soto y Conde (1990).
* A. caespitosa (J. Agardh) Dixon = Acrochaetium caespitosum $(\mathrm{J}$. Agardh) Näg.

a) 9, 11 y 18. b) PhI. c) Epífita de Cystoseira sp. pl. y Pterocladia capillacea. d) Ab.

A. codii (H. M. et P. L. Crouan) Garbary $=$ Acrochaetium codii Born.

a) 4, 8, 9 y 26. b) PhI. c) Epífita de Codium grex dichotomus, exceptuando en el interior de la Mar Chica donde se ha encontrado sobre Sargassum vulgare. d) Ab.

* A. corymbifera (Thur. in Le Jolis) Dixon = Acrochaetium corymbiferum (Thur. in Le Jolis) Batters

a) 3 y 14. c) Epífita de Zostera noltii y Pterocladia capillacea.

A. daviesii (Dillwyn) Woelkerling = Acrochaetium daviesii (Dillwyn) Näg. d) APo

a) MD. b) ISR. c) Epífita de varias especies.

* A. microscopica (Näg. in Kütz.) Woelkerling

= Acrochaetium microscopicum Näg.

a) 11 y 27. b) RM. c) Epífita de Centroceras clavulatum, Halopteris filicina y Cladostephus spongiosus. d) APtf.

A. moniliformis (Rosenvinge) Garbary

= Acrochaetium moniliforme (Rosenvinge) Boerg.

a) 5 y 9. b) ISR. c) Epífita de Cladophora sp. y Halopteris filicina. d) M.

A. parvula (Kylin) Dixon

= Acrochaetium parvulum (Kylin) Hoyt

a) MD. b) PhI. c) Epífita de Cladoforáceas y Ulváceas en la Mar Chica y en el resto del litoral de Pterocladia capillacea, Cymodocea nodosa, Gelidium grex pusillum, Corallina sp.pl., Zonaria tournefortii, Cystoseira sp.pl., Sargassum vulgare y Codium sp.pl. d) Ab.

A. saviana (Menegh.) Woelkerling = Acrochaetium savianum (Menegh.) Näg.

a) MD. b) PhI. c) Epífita de Codium grex dichotomus, Sargassum sp. pl. y Cystoseira sp. pl. d) Abt. 
A. cf. spetsbergensis (Kjellm.) Woelkerling = Rodochorton spetsbergensis (Kjellm.) Kjellm.

a) 34. b) RMMI. c) Epífita de Rissoella verruculosa. d) M.

* A. subpinnata (Born. ex Hamel) Garbary = Acrochaetium subpinnatum Born. ex Hamel a) 8 y 19. b) PhIC. c) Epífita deCladophora sp.pl., y Cladostephus spongiosus. d) Ab.

* A. thuretii (Born.) Woelkerling

= Acrochaetium thuretii (Born.) Collins et Harv.

a) 27 y 28. b) SSB. c) Epífita de Gelidium sesquipedale y Pterocladia capillacea. d) APo.

* A. trifila (Buffham) Dixon

= Acrochaetium trifilum (Buffham) Batters

a) 27. b) RM. c) Epífita de Cladophora dalmatica. d) Ab.

Schmitziella endophloea Born. et Batters ex Batters

a) 2, 3, 4, 9, 15, 19, 24 y 27. b) AS. c) Epífita de Cladophora prolifera .d) C.

\section{GELIDIALES}

*Gelidium crinale (Turner) Lamour.

a) MD. b) FM. d) APo.

G. latifolium (Grev.) Born. et Thur.

a) MD. b) PhI. c) Frecuentemente forófita de Cladoforáceas, Ceramiáceas, Audouinella sp. pl. y Erythrotrichia sp. pl. d) SC.

G. latifolium var. histrix (J. Agardh) J. Feldm. et Hamel
a) $9,12,14,18,20,24,27$ y 34 . b) PhI. d) SC.

* G. melanoideum Schousb. ex Born.
a) 3. b) SSB. c) M.

G. pusillum (Stackh.) Le Jolis

a) MD. b) PhI y FM. d) C.

* G. sesquipedale (Clemente) Born. et Thur.

a) 27 y 28 . b) SSB. d) Ab.

* G. spathulatum (Kütz.) Born.
a) 2, 3, 8, 15, 18, 21 y 27. b) PhIC. d) Ab.

Pterocladia capillacea (S.G. Gmelin) Born. et Thur. $=P$. pinnata (Huds.) Papenfuss

a) MD. b) SSB. d) SC. e) Forófita de Titanoderma pustullatum.

Gelidiella lubrica (Kütz.) J. Feldm. et Hamel
a) 3, 5, 9, 12 y 34 . b) PhIC. d) M.

* G. nigrescens (J. Feldm.) J. Feldm. et Hamel

$$
\text { a) 3,10,11 y 14. b) SI. d) M. }
$$

\section{NEMALIALES}

Nemalion helminthoides (Velley in With.) Batters a) $2,3,9,13,14,16,17,23,27$ y 34 . b) RMM1. d) SC. e) Frecuentemente asociada a Lithophyllum lichenoides.

* Helminthocladia stackhousei (Clemente)
Cremades
$\quad=$ Helminthora divaricata (C. Agardh) J. Agardh

a) 15. d) Ab.

* Liagora distenta (G. Mertens ex Roth) C. Agardh a) $3,15,18,19,20,24,26$ y 28 . b) PhIC. d) Ao.

* L. viscida (Forsskaal) C. Agardh

a) $3,15,18,19,20,24,26$ y 28. b) PhIC. d) IAo. e) En comunidades con Amphiroa rigida, Liagora distenta y Dictyopteris polypodioides.

* Scinaia furcellata (Turner) J. Agardh $=S$. forcellata Biv. Bern.

a) 27. d) APo.

BONNEMAISONIALES

Asparagopsis armata Harv.

a) MD. b) ISR. d) C. e) Incluye Falkenbergia rufolanosa (Harv.) F. Schmitz.

*Trailliella intricata Batters

a) 13. c) Epífita de Halopteris scoparia. d) Ab. e) Tetrasporófito de Bonnemaisonia clavata Hamel. 


\section{GIGARTINALES}

Calliblepharis ciliata (Huds.) Kütz.
a) 33. b) SM. d) Ab.

Hypnea musciformis (Wulfen) Lamour.

a) MD. b) PhIT. d) P.

Rissoëlla verruculosa (Bertol.) J. Agardh

a) 28 y 34. b) RMM1. d) M.

Sphaeroccocus coronopifolius Stackh.

a) $1,2,3,10,11,13,14,18,20,28$ y 34 . b)

SCI. c) Forófita de Hypoglossum hypoglossoides, Acrosorium venulosum, Erythrotrichia carnea, Stylonema alsidii, Ceramium flaccidum y Erythropeltis subintegra. d) Ab.

Plocamium cartilagineum (L.) Dixon $=P$. coccineum (Huds.) Lyngb.

a) MD. b) SSBf. d) SC.

Gracilaria armata (C. Agardh) J. Agardh

a) 5 y 27. b) PhIB. c) Forófita de Ceramium sp. pl. y Cladophora lehmaniana. d)At.

G. bursa-pastoris (S.G. Gmelin) Silva $=$ G. compressa $($ C. Agardh $)$ Grev.

a) 7, 22, 27. b) PhI. d) SC.

G. cervicornis (Turner) J. Agardh

a) 5. b) PhI. d) At.

* G. foliifera (Forsskaal) Boerg.

$=$ G. multipartita (Clemente) J. Agardh

a) $3,18,22$. b) SI. c)f Forófita de Pneophyllum

lejolisii, Pterosiphonia complanata y Audouinella saviana. d) IAo.

G. verrucosa (Huds.) Papenfuss

$=G$. confervoides $(\mathrm{L}$.$) Grev.$

a) 2, 5, 6, 7 y 8. b) PhI. d) C.

* Gymnogongrus crenulatus (Turner) J. Agardh $=G$. norvegicus (Turner) J. Agardh

a) MD b) SSBf. c) Forófita de Aphanocladia stichidiosa. d) Ab y M.

G. griffithsiae (Turner) Mart.

a) 5, 21, 22, 23. b) FM. d) CB.
Phyllophora crispa (Huds.) Dixon $=P$. nervosa $(\mathrm{D} \mathrm{C})$ Grev.; P. epiphylla $(\mathrm{O} . \mathrm{F}$. Müll.) Batters; P. rubens (L.) Grev.

a) 2, 3, 4, 19, 20, 22, 23 y 27. b) SI. c) Forófita de Hypoglossum hypoglossoides, Pterothamnion crispum y Jania rubens. d) Ab.

* P. sicula (Kütz.) Guiry et L. Irvine $=P$. palmettoides J. Agardh

a) 18 y 27. b) SSB. d) Ab.

Schottera nicaensis (Lamour. ex Duby) Guiry et Hollenberg

Schotter

= Petroglossum nicaense (Lamour. ex Duby)

a) $3,4,9,11,12,17$ a 24,27 y 28 . b) SSB. c) Epífita de Halopteris filicina, Peyssonnelia squamaria, Corallina elongata, Lithophyllum dentatum, Amphiroa beauvoisii, Cladostephus spongiosus, Dictyopteris polypodioides y Codium bursa; forófita de Acrosorium venulosum y Ceramium flaccidum. d) Ab.

Gigartina acicularis (Roth) Lamour.

a) MD. b) PhIP. c) A veces epífita de Cystoseira tamariscifolia y Corallina elongata.; forófita de Sphacelaria cirrosa. d) C.

* G. pistillata (S.G. Gmelin) Stackh. a) $19,20,22,23,27$ y 28. b) SSB. d) Ab.

\section{CORALLINALES}

Amphiroa beauvoisii Lamour.

a) 1, 2, 6, 8, 9, 11, 12, 18, 20, 27 y 34. b) SC. c) Forófita de Audouinella daviesii, Champia parvula, Acrosorium venulosum, Schottera nicaensis, Aphanocladia stichidiosa, Callithamnion granulatum, Sphacelaria rigidula, Stylonema sp.pl. y Erythrotrichia sp.pl. d) IAt.

A. cryptarthrodia Zanardini

a) 3, 8, 9, 20, 22, 27 y 34. b) SC. c) Forófita de Acrosorium venulosum, Crouania attenuata, Pterosiphonia complanata y Aglaothamnion byssoides. d) APt.

* A. fragilissima (L.) Lamour.

a) 12 y19. b) SI. c) Epífita de Peyssonnelia squamaria. d) M. 
A. rigida Lamour.

a) 1, 2, 3, 12, 15, 18, 19, 20, 21, 22, 25, 28 y 34. b) PhI. c) Epífita de Mesophyllum lichenoides y Lithophyllum dentatum. d) SC.

Choreonema thuretii (Born.) F. Schmitz

a) 1, 3, 9, 17, 22, 25, 27 y 32. b) PhI. c) Epífita de Jania rubens, Jania longifurca y Corallina elongata. d) C.

\section{* Corallina attenuata Kütz.}

a) 3 y 15. b) PhIC a $2 \mathrm{~m}$ de profundidad. d) M. e) Para separar esta especie de $C$. granifera nos hemos basado en Bressan (1974).

C. elongata J. Ellis et Sol.

$=C$. mediterranea Areschoug in J. Agardh

a) MD. b) ISR. c) Forófita de Ceramiáceas, Ectocarpáceas, Audouinella sp.pl.y Erythrotrichia sp. pl., entre otros. d)Ab.

C. granifera J. Ellis et Sol.

$=C$. virgata Zanardini; Haliptilon virgata (Zanardini) Garbary et Johansen

a) $2,9,10,13,16,17,28$ y 34 . b) PhIT. c) Epífita de Cystoseira tamariscifolia y $C$. mediterranea. d) IAtf.

\section{C. officinalis $\mathrm{L}$.}

a) $1,2,3,10,13,14,16,17,21,22,23,25,27$, 28, 30, 32 y 34. b) PhIT. c) Forófita de Ceramium diaphanum, Cottoniella filamentosa, Boergeseniella fruticulosa, Ectocarpus fasciculatus y Audouinella daviesii. d) APo.

Fosliella farinosa (Lamour.) M. Howe in Britton et Millsp. = Melobesia farinosa Lamour.

a) MD. b) SC. c) Epífita de Sargassum sp.pl., Codium bursa, Valonia utricularis., Zostera marina y Cymodocea nodosa. d) C.

* Goniolithon papillosum (Zanardini ex Hauck) Foslie

= Lithothamnion papillosum Zanardini ex Hauck; Lithophyllum papillosum (Zanardini ex Hauck) Huvé; Dermatolithon papillosum (Zanardini) Foslie
a) 4 y 15 . b) EM. d) M.

Jania adhaerens Lamour.

a) 34. b) PhIT. d) IP.

J. corniculata (L.) Lamour.

a) $9,10,11,13,16,17,20,21,23,24,27,28$ 32 y 34. b) PhIC. c) Epífita de Halopteris scoparia, H. filicina, Cystoseira tamariscifolia, Sargassum vulgare y Cladostephus spongiosus. d) Ab.

\section{J. longifurca Zanardini}

a) 3, 9, 16, 17, 18, 22, 23, 27, 28 y 34. b) PhIB.

c) Epífita de Cystoseira tamariscifolia. d) Ab.

J. rubens (L.) Lamour.

a) MD. b) PhI. c) Epífita de Halopteris filicina y H. scoparia . d) C.

Lithophyllum expansum Phil. sensu Lemoine = Pseudolithophyllum expansum (Phil.) Lemoine

a) $1,9,10,11,12,13,15,18,20,22,23,27$, $30,31,32$ y 33 . b) SC. d) At.

L. incrustans Phil.

a) MD. b) ISR. d) Ab. e) Es característica su presencia en zonas de abundancia en equinodermos de la especie Paracentrotus lividus (LithophylloArbacietum lixulae).

* L. lichenoides Phil.

$=$ L. tortuosum auct. sensu Lemoine; Tenarea tortuosa (Esper) Lemoine

a) $2,3,9,13,14,16,17,18,22,23,27$ у 28 .

b) EM. d) CB.

* Melobesia membranacea (Esper) Lamour. = Epilithon membranaceum (Esper) Heydr.

a) 1, 10, 12, 19, 22, 25 y 27. b) SSB. c) Epífita de Pterocladia capillacea, Corallina elongata, Amphiroa beauvoisii, Cladophora coelothrix y Cystoseira tamariscifolia. d) IAo.

* Mesophyllum lichenoides (J. Ellis) Lemoine a) $1,3,9,10,11,12,13,14,18,21,22,23,27$ y 34. b) SIC. c) Forófita de Ceramiáceas. d) Abt.

* Pneophyllum lejolisii (Rosanoff) Chamberlain = Melobesia lejolisii Rosanoff; Fosliella lejolisii (Rosanoff) M. Howe a) 3, 20, 27 y 34 . b) HP. d) C. 
* Spongites dentata Kütz.

$=$ Lithophyllum dentatum (Kütz.) Foslie

a) $3,8,15,18,19,20,21,22,23,24,25,27$, 28 y 34. b) PhIC c) Forófita de Jania rubens, Halopteris filicina, Amphiroa beauvoisii, A. rigida, Ectocarpáceas, Heterosiphonia crispella y algunas Ceramiáceas. d) Ab.

S. fruticulosa Kütz.

$=$ Lithothamnion fruticulosum (Kütz.) Foslie a) 4, 5, 6, 7, 21 y 25. b) SM. c) Ab.

* S. notarisii (L. Dufour) Athanasiadis

= Melobesia notarisii L. Dufour; Neogoniolithon notarisii (L. Dufour) Setch. et Mason
a) $13,21,27$ y 28 . b) PhI. d) Ab.

* Titanoderma cystoseirae (Hauck) Woelkerling, Chamberlain et Silva

$=$ Lithophyllum cystoseirae (Hauck) Heydr.

a) $10,15,16,17,21,22,23,24$ y 27. c) Epífita de Cystoseira tamariscifolia y $C$. mediterranea. $\mathrm{d}$ ) Ab.

T. mediterranea (Foslie) Woelkerling

= Litholepis mediterranea Foslie

a) 2, 3, 11, 21 y 31. b) Epífita de Cystoseira sp. pl. y Sargassum vulgare. d) Ab.

T. pustullatum (Lamour.) Näg.

= Melobesia pustulata Lamour; Dermatolithon pustulatum (Lamour.) Foslie; Lithophyllum pustulatum (Solms) Lemoine

a) MD. b) ISR. c) Epífita de Pterocladia capillacea y también sobre moluscos como Thais haemastoma, Mytilus sp. o Pinna nobilis. d) C.

\section{CRYPTONEMIALES}

Grateloupia filicina (Lamour.) C. Agardh

a) $5,9,12,21,23$ y 27. b) SSBc. d) SC.

* Halymenia floresia (Clemente) C. Agardh a) 15,20 y 22. b) CC. d) SC.

* Peyssonnelia atropurpurea H. M. et P. L. Crouan a) 11,12 y 13. b) SC. c) Forófita deTaenioma nanum y Acrosorium venulosum. d) Ab.
P. bornetii Boudour. et Denizot

a) 4,13 y 27 . b) SC. d) M.

P. coriacea J. Feldm.

a) MD. b) SC. c) Forófita de Schottera nicaensis, Acrosorium venulosum, Apoglossum ruscifolium, Hypoglossum hypoglossoides, Jania rubens, Ceramium sp.pl. y Audouinella sp.pl. d) M.

P. dubyi H. M. et P. L. Crouan

= Cruoriella dubyi (Crouan) F. Schmitz

a) $3,4,5,15,19,22,24,25$ y 28 . b) ISR. d) $\mathrm{Ab}$.

* P. rubra (Grev.) J. Agardh

a) 11,12 y 34. b) SC. c) Epífita en los rizomas de Posidonia oceanica; forófita de Herposiphonia secunda y Audouinella saviana. d) Ab).

\section{P. squamaria (S.G. Gmelin) Decne.}

a) MD. b) SCIT. c) Forófita de Ceramium diaphanum, Erythrotrichia carnea, Stylonema sp.pl., Erythropeltis subintegra, Audouinella daviesii, A. saviana, A. parvula, Centroceras clavulatum y Dasya baillouviana. d) M.

\section{RHODYMENIALES}

Champia parvula (C. Agardh) Harv.

a) $2,3,8,9,11,14,15,18,21,24,26,2830$ y 34. b) SC. c) Epífita de Gigartina acicularis e Hypnea musciformis; forófita de Ceramium tenerrimum, Dasya rigidula, Heterosiphonia crispella y Audouinella daviesii. d) C.

* Chylocladia verticillata (Lightf.) Bliding

$=$ Ch. kaliformis (Good. et Woodw. ) Grev; Lomentaria kaliformis Gaillon

a) 15 y 18 . b) PhIC y IAtf.

Gastroclonium clavatum (Roth) Ardiss.

a) $1,4,9,11,14,15,21,22,25,27$ y 34 . b) EM e infralitoral superior. c) Epífita de Cystoseira compressa, $C$. tamariscifolia, Corallina elongata e Hypnea musciformis; forófita de Centroceras clavulatum y Heterosiphonia crispella. d) M.

Lomentaria clavellosa (Turner) Gaillon var. conferta (Menegh.) J. Feldm.

a) 34. b) SI. y IAtf. 
* L. clavellosa var. reducta Ercegovic

a) 8,15 y 25. b) SI. c) Epífita de Amphiroa beauvoisi, Codium tomentosum y Corallina elongata. d) IAtf.

* L. firma (J. Agardh) Kylin

a) 8 . b) M.

* Botryocladia botryoides (Wulfen) J. Feldm. $=$ Chrysymenia uvaria $(\mathrm{C}$. Agardh) $\mathrm{J}$. Agardh a) 3, 11 y 27. b) SSBc. d) Abt.

* Cordylecladia erecta (Grev.) J. Agardh

a) 3. b) En grandes cubetas litorales de menos de $1 \mathrm{~m}$ de profundidad. d) Ab.

\section{CERAMIALES}

Aglaothamnion byssoides (Arnott ex Harv. in Hook.) L'Hardy-Halos \& Rueness Hook.

= Callithamnion byssoides Arnott ex Harv. in

a) $3,5,8,9,11,15,19,24$ у 27 . b) SI. c) Epífita de Cladostephus spongiosus, Halopteris filicina, Cystoseira crinita, Colpomenia sinuosa, Acetabularia acetabulum y Amphiroa cryptarthrodia. d) Abt.

* A. hookeri (Dillwyn) Maggs \& Hommersand = Callithamnion hookeri (Dillwyn) S.F. Gray

a) 27. b) PhIC. c) Epífita de Sargassum vulgare. d) Ab.

A. neglectum Feldm.-Mazoyer

= Callithamnion neglectum (Feldm.-Mazoyer)

Ballesteros \& Romero

a) 3, 4, 5, 8, 9, 18 y 26. b) SC. c) Epífita de Codium fragile, C. vermilara y Gelidium pusillum. d) At.

Anotrichum tenue (C. Agardh) Näg.

$=$ Griffthsia tenuis $\mathrm{C}$. Agardh

a) 4, 5, 8, 9 y 15. b) SI. c) Epífita de Hypnea musciformis, Corallina attenuata, Pterocladia capillacea y Amphiroa beauvoisii. d) IP.

* Antithamnion algeriensis Verlaque et Seridi

a) $3,9,12,18,20$ y 27 . c) Epífita de coralináceas ramificadas, Gelidium latifolium y Pterocladia capillacea e) Aunque no se han detec- tado la presencia de pelos hialinos ni órganos reproductores, los ejemplares recolectados se ajustan a lo descrito por Verlaque \& Seridi (1991) en las costas argelinas.

A. cruciatum (C. Agardh) Näg.

a) $3,4,5,8,9,11,12,14,16,18,22,23,25$ y

27. b) ISR c) Epífita de Peyssonelia coriacea, Gelidium latifolium, Lithophyllum dentatum, Corallina elongata, Plocamium cartilagineum y epizoica sobre hidrozoos y ascidiarios. d) Abt.

Antithamnionella elegans (Berthold) Boudour. et Verlaque

a) $2,3,4,5,6,8,9,11,12,19,27$ у 28 . b) SSBf. c) Epífita de Gelidium latifolium, Plocamium cartilagineum, Corallina elongata, Pterocladia capillacea, Aphanocladia stichidiosa, Dictyopteris polypodioides, Amphiroa beauvoisii, Sargassum vulgare, Polysiphonia fucoides, Centroceras clavulatum, Hypnea musciformis y Ulva olivascens. d) $\mathrm{M}$.

* A. spirographidis (Schiffn.) Wollaston = Antithamnion spirographidis Schiffn.

a) 11. b) HSPP. c) Epífita de Peyssonnelia coriacea. d) IAtf.

* Callithamniella tingitana (Schousb. ex Born.) Feldm.-Mazoyer

a) 8, 9 y 24. b) PhIG. c) Epífita de Acrosorium venulosum y Peyssonnelia squamaria. d) M.

* Callithamnion corymbosum (Sm.) Lyngb.

a) $1,3,9,10,11,12,16,17,19,20,23$ y 27. b) SIC. c) Epífita de Cystoseira tamariscifolia, Pterocladia capillacea, Gelidium pusillum, Laurencia papillosa, L. undulata y Chondria capillaris. d) Abt.

C. granulatum (Ducluz.) J. Agardh

a) $3,9,11,19,21,22,27,32$ y 34. b) RMM1. c) Saxícola o epífita de Lithophyllum lichenoides, Corallina elongata y Pterocladia capillacea. d) Ab.

C. tetragonum (With.) S.F. Gray

a) $11,12,18,19,22,27,28$ y 34. b) SSBf. c) Epífita de Colpomenia sinuosa, Pterocladia capillacea, Gymnogongrus crenulatus y Codium 
decorticatum. d) Ab.

Centroceras clavulatum Mont.

a) $2,4,5,6,8,9,10,15,18,19,21,24,25,26$ y 27. b) PhIP. c) Epífita de Spyridia filamentosa, Derbesia neglecta, Cladostephus spongiosus, Cladophora prolifera, Cl. lehmaniana, Alsidium corallinum; forófita de Audouinella parvula, Erythrotrichia carnea y Ceramium tenerrimum. d) C.

Ceramium ciliatum (J. Ellis) Ducluz.

a) 22, 28 y 34. b) FM. d) Ab.

C. ciliatum var. robustum (J. Agardh) G. Mazoyer a) $2,9,10,11,12,15,18,19,20,22,23,27$, 28 y 34. b) FM. c) Epífita de Ulva rigida, Gracilaria armata, Gelidium sesquipedale, Chondria capillaris, Gelidium latifolium y Colpomenia sinuosa. d) M.

* C. cinnabarinum (Grateloup ex J. Agardh) Hauck = Ceramium ordinatum Kütz; Centroceras cinnabarinum (Grateloup) J. Agardh

a) 17. b) PhIB. c) Epífita de Aphanocladia stichidiosa y Gelidium latifolium. d) M.

C. cingulatum A. Weber

a) 3, 4, 5, 12, 13, 20 y 27. c) Epífita de Zostera noltii, Chondria mairei, Sargassum vulgare y Halopteris filicina.

* C. circinatum (Kütz.) J. Agardh

a) 27. c) Epífita de Gelidium pusillum. d) Atf.

* C. codii (Richards) G. Mazoyer

a) 3, 4, 8, 9 y 12. b) SC. c) Epífita de Acetabularia acetabulum, Polyphysa parvula, Cladophora prolifera y Heterosiphonia crispella. d) IAo.

C. diaphanum (Lightf.) Roth

a) MD. b) ISR. c) Epífita de Sargassum vulgare, Cystoseira sp.pl., Codium sp. pl., Padina pavonica, Gracilaria verrucosa, Zostera marina, Z. noltii, Cymodocea nodosa y sobre moluscos (Patella sp., Thais haemastoma). d) SC.

* C. diaphanum var. lophophorum Feldm.Mazoyer a) 11 y 27. b) CC. c) Epífita de Sphaeroccocus coronopifolius. d) At.

C. echionotum J. Agardh

a) $11,12,18,23,27,28,32$ y 34 . b) SSBf. c) Epífita de Cystoseira tamariscifolia, Halopteris filicina y Gelidium pusillum. d) Ab.

C. flaccidum (Harv. ex Kutz.) Ardiss.

$=C$. gracillimum Harv.

a) $1,2,3,4,5,7,8,9,10,13,18,20,22,23$, 27, 28 y 34. b) ISR. c) Epífita de Amphiroa sp.pl., Plocamium cartilagineum, Cymodocea nodosa, Zostera marina, Boergeseniella fruticulosa, Sargassum sp. pl., Mesophyllum lichenoides, Halopytis incurvus y Cystoseira crinita. d) SC.

C. nodosum (Kutz.) Harv.

= C. tenuissimum (Roth) J. Agardh

a) $3,4,5,7,9,12,15$ y 34. b) PhIC. c) Epífita

de Cystoseira crinita, Liagora distenta, Acetabularia acetabulum, Cystoseira compressa y Zostera marina. d) SC.

C. nodulosum (Lightf.) Ducluz.

$=$ C. rubrum (Huds.) C. Agardh

a) MD. b) PhIB. c) Saxicola y epífita de Cystoseira sp.pl., Codium tomentosum, Laurencia pinnatifida, Colpomenia sinuosa, Sargassum sp.pl. d) APo.

* C. nodulosum var. barbatum (Kütz.) J. Agardh a) 12,19 y 27. c) Epífita de Cystoseira compressa, C. tamariscifolia y Laurencia pinnatifida. d) Ab.

* C. nodulosum var. implexo-contortum Solier

a) 9,21, 22, 27 y 28. c) Epífita de Corallina elongata, Pterocladia capillacea y Cystoseira tamariscifolia. d) M.

C. nodulosum var. tenue C. Agardh

a) 2, 3 y 34. c) Epífita de Laurencia pinnatifida, Corallina elongata y Cladophora lehmanniana. d) M.

C. tenerrimum (Martens) Okamura

a) $3,4,5,8,9,10,11,12,15,19,27,28$ у 34 . b) PhI. c) Epífita de Centroceras clavulatum, Padina pavonica, Amphiroa beauvoisii, Cymodocea 
nodosa, Dasya hutchinsiae, Zostera marina, Boergeseniella fruticulosa y Alsidium corallinum. d) SC.

* Compsothamnion thuyoides (Sm.) Näg. = Callithamnion thuyoides $(\mathrm{Sm}$.$) C. Agardh;$ C. gracillimum C. Agardh

a) 11,14 y 24. b) HSPP. c) Epífita de Peyssonnelia coriacea y Cystoseira elegans. d) Abt.

Crouania attenuata (Bonnem. ex C. Agardh) J. Agardh

a) $1,3,9,11,13,15,18,20,21,25,27,28 \mathrm{y}$ 34. b) PhI. c) Epífita de Gelidium latifolium var. hystrix, G. pusillum, Lithophyllum dentatum, Dasya rigidula, D. hutchinsiae, Halopytis incurvus, Cladostephus spongiosus, Halopteris filicina, Plocamium cartilagineum, Seirospora gyraudii, Cystoseira mediterranea y Boergeseniella thuyoides. d) SC.

* Dohrniella neapolitana Funk

a) 5. c) Epífita de Grateloupia filicina. d) M.

Griffithsia opuntioides J. Agardh

a) 3, 27, 28 y 34. b) SSB. c) Epífita de Pterocladia capillacea y Lithophyllum lichenoides. d) Ab.

* G. phyllamphora J. Agardh

a) $11,12,18,19$ y 20. b) SSBc. c) Epífita de Amphiroa cryptarthrodia, Cladophora pellucida y Schottera nicaensis. d) M.

* G. schousboei Mont. Abt.

a) 11. b) SCIT. c) Epífita de Amphiroa sp. d)

* Gymnothamnion elegans (Schousb. ex C. Agardh) J. Agardh

a) 8, 12 y 20. b) SSB. c) Epífita de Pterocladia capillacea y Champia parvula. d) APo.

* Halurus flosculosus (J. Ellis) Maggs \& Hommersand Newton

= Griffithsia flosculosa (J. Ellis) Batters ex

a) 27. b) SI. c) Epífita de Schottera nicaensis.
* Lejolisia mediterranea Born.

a) 27. b) SSB c) Epífita de Codium decorticatum y Pterocladia capillacea. d) P.

Monosporus pedicellatus ( $\mathrm{Sm}$.) Solier in Castagne var. tenuis Feldm.-Mazoyer

= Corynospora pedicellata $(\mathrm{Sm}.) \mathrm{J}$. Agardh; Monospora pedicellata (Sm.) J. Agardh; Neomonospora pedicellata (Sm.) Feldm.-Mazoyer \& Meslin

a) 5. b) SIC. c) Epífita de Cystoseira crinita y C. compressa. d) M.

* Pleonosporium borreri (Sm.) Näg. ex Hauck

a) 8,11 y 27. b) SI. c) Epífita de Amphiroa rigida, Dictyota dichotoma y Cystoseira compressa. d) Abt.

* Pterothamnion crispum (Ducluz.) Näg.

= Callithamnion plumula (J. Ellis) Lyngb. var. crispum (Ducluz.) J. Agardh; Platythamnion crispum (Ducluz.) J. Feldm.

a) 18,20 y 27. b) SCI. c) Epífita de Phyllophora crispa, Zonaria tournefortii, Acrosorium venulosum y Bryopsis hypnoides. d) SC.

* Pt. plumula (J. Ellis) Näg.

= Callithamnion plumula (J. Ellis) Lyngb; Antithamnion plumula (J. Ellis) Thur. ex Le Jolis a) 2, 8, 10, 11, 15, 19 y 27. b) SCI. c) Epífita de Zonaria tournefortii, Dictyota dichotoma y Plocamium cartilagineum. d) SC.

* Pt. plumula var. bebii (Reinsch) Cormaci et Furnari

a) $1,9,11,12,15,21,22,24$ y 27 . b) SC. c) Epífita de Cystoseira schiffneri, Dictyopteris polypodioides, Cladophora prolifera, Plocamium cartilagineum, Dictyota dichotoma var. implexa. d) SC.

* Ptilothamnion pluma (Dillwyn) Thur. in Le Jolis = Callithamnion pluma (Dillwyn) C. Agardh a) 11 y 15.b) SC. c) Epífita de Heterosiphonia crispella y Zonaria tournefortii. d) Ab.

* Seirospora giraudyi (Kütz.) De Toni

a) 27. b) SI. c) Epífita de Gigartina pistillata. d) $\mathrm{M}$.

d) Abt. 
* Spermothamnion flabellatum Born. et Thur.

a) $2,8,11,15$ y 27. b) SCI. c) Epífita de Codium grex dichotomus, Lophosiphonia cristata y Cystoseira spinosa. d) M.

* S. johannis Feldm.-Mazoyer

a) 10. b) CC. c) Epífita de hidrozoos en una comunidad dominada por Peyssonnelia squamaria y Lithophyllum incrustans. d) M.

Spyridia filamentosa (Wulfen) Harv. in Hook.

a) $3,4,5,6,7,15,18,23,27$ y 34. b) PhIT.

c) Epífita de Padina pavonica, Pterocladia capillacea y Laurencia pinnatifida; forófita de Callithamnion granulatum, Enteromorpha multiramosa y Cladoforáceas. d) C.

* Vickersia baccata (J. Agardh) Karsakoff a) 3. b) SI. d) Ab.

Dasya elegans (Martens) C. Agardh $=D$. baillouviana (S.G. Gmelin) Mont.

a) 4, 5, 8 y 18. b) RMM1. c) Epífita de Corallina attenuata y Gelidium pusillum. d) IAo.

* D. hutchinsiae Harv. in Hook.

= D. arbuscula Harv.

a) $1,3,9,11,13,15,22$ y 28. c) Epífita de Boergeseniella fruticulosa, B. thuyoides, Dilophus spiralis, Cystoseira tamariscifolia, Codium decorticatum, C. elongatum y Ceramium nodulosum; forófita de Crouania attenuata. d) Ab.

* D. ocellata (Grateloup) Harv. in Hook.

a) 8, 24. b) SIC. c) Epífita de Peyssonnelia squamaria y Zonaria tournefortii. d) Abt.

D. rigidula (Kütz.) Ardiss.

a) 3, 18, 27 y 34. b) PhIC. c) Epífita de Cymodocea nodosa y Halopteris scoparia. d) Abt.

* Dasyopsis plana (C. Agardh) Zanardini

a) 11. b) CCT. c) Epífita de Peyssonnelia squamaria. d) Ab.

Heterosiphonia crispella (C. Agardh) Wynne $=H$. wurdemannii (Bailey) Falkenb.

a) MD. b) SI c) Epífita de Dictyota dichotoma, Cladostephus spongiosus, Plocamium cartilagineum, Lithophyllum incrustans,
Peyssonnelia squamaria, Jania longifurca, Dilophus spiralis, Halopytis incurvus, Corallina elongata, Amphiroa beauvoisii, Codium tomentosum y Dictyopteris polypodioides. d) SC.

H. plumosa (J. Ellis) Batters

= Dasya coccinea (Huds.) C. Agardh

a) 33 .

Acrosorium venulosum (Zanardini ex Kütz.) Kylin $=A$. uncinatum (Turner) Kylin; $A$. uncinatum var. reptans (H. M. et P. L. Crouan) Boudour. et al. a) MD. b) SI. c) Epífita de Phyllariopsis brevipes,Sargassum vulgare, Dictyopteris polypodioides, Cladophora prolifera, Pterocladia capillacea, Sphaerococcus coronopifolius, Peyssonnelia coriacea, Phyllophora crispa y Halopytis incurvus; forófita de Pterothamnion crispum y Audouinella saviana . d) C.

Apoglossum ruscifolium (Turner) J. Agardh

a) $2,3,11,14,17,18,22,23,25,27,28$ у 34 . b) SIC. c) Epífita de Sphaerococcus coronopifolius, Phyllophora crispa, Phyllariopsis brevipes, Halopteris scoparia, Dictyopteris polypodioides, Dilophus sp. pl. y Zonaria tournefortii. d) Abt.

* Cottoniella filamentosa (M. Howe) Boerg.

a) $2,9,10,11,12,14,18,20,22$ y 24 . b) Franja litoral o infralitoral superior. c) Frecuentemente epifitando o enredada a Pterocladia capillacea y Cystoseira sp.pl. d) IAt.

* Cryptopleura ramosa (Huds.) Kylin ex Newton

a) 18,19 y 27 . b) Litoral e infralitoral y en cubetas litorales. c) Epífita de Cystoseira compressa y Halopteris filicina; forófita de Erytrhrotrichia carnea, Stylonema sp. pl. y Audouinella daviesii. d) Abt.

* Haraldia lenormandii (Derbès et Solier in Castagne) J. Feldm.

a) 17. b) SI. c) Epífita de Peyssonnelia squamaria. d) Abt.

Hypoglossum hypoglossoides (Stackh.) Collins et Hervey

$=H$. woodwardii Kütz.

a) MD. b) SIC. c) Epífita de Sphaerococcus coronopifolius, Phyllophora crispa, Codium 
decorticatum, Cladostephus spongiosus, Scytosiphon simplicissimus, Peyssonnelia sp.pl. e Hypnea musciformis. d) Ab.

\section{* Myriogramme gaiolae Funk}

a) 27. b) SSB. c) Epífita de Jania longifurca. d) M. e) La estructura del fronde y la disposición de los soros coincide con los datos de Funk (1955).

* Nithophyllum albidum Ardiss. $=N$. charybdaeum Borzi

a) 12. b) PhIC. c) Epífita de Corallina elongata y forófita de Pterosiphonia pennata. d) M.

\section{* Taenioma nanum (Kütz.) Papenfuss}

a) $4,5,9,10,12$ y 18. b) Medio e infralitoral superior, en cubetas y plataformas de poca hidrodinamia. c) Epífita de Hypnea musciformis. d) Ab.

\section{Alsidium corallinum C. Agardh}

a) 1, 3, 4, 5, 6, 7, 9 y 18. b) PhIT. c) Forófita de Erythrotrichia carnea, Ceramium sp.pl., Ulváceas y Cladoforáceas. d) At.

Aphanocladia stichidiosa (Funk) Ardré

a) MD. b) SI. c) Epífita de Cystoseira tamariscifolia, C. mediterranea, Pterocladia capillacea, Pterosiphonia pennata, Coralináceas y Gelidium pusillum. d) M.

Boergeseniella fruticulosa (Wulfen) Kylin = Polysiphonia fruticulosa (Wulfen) Spreng.

a) MD. b) PhIB. c) Epífita de Cystoseira tamariscifolia. d) Ab.

* B. thuyoides (Harv. in MacKay) Kylin = Polysiphonia thuyoides Harv. in MacKay; Pterosiphonia thuyoides (Harv. in MacKay) Batters a) $12,13,16,17,18,20,22,23,27$ y 28 . b) PhIB. c) Epífita de Cystoseira tamariscifolia. d) $\mathrm{Ab}$.

Chondria capillaris (Huds.) Wynne $=$ Ch. tenuissima (With. ) C. Agardh

a) 2, 3, 4, 5, 7, 9, 11, 15, 18, 19, 22, 27 у 34 . b) PhIC. c) Epífita de Zostera marina Z. noltii y Cymodocea nodosa; forófita de Ceramium cingulatum, C. diaphanum, Erythropeltis subintegra, Audoinella daviesii y A. parvula. e) IAo.

* Ch. coerulescens (J. Agardh) Falkenb.

a) $2,3,5,9,10,15,20,22$ y 27. b) Litoral e infralitoral superior. c) Forófita de Cladophora dalmatica, Audouinella parvula, A. daviesii, Erythrotrichia carnea y Stylonema sp.pl. d) Ab.

Ch. dasyphylla (Woodw. ) C. Agardh

a) 4, 5, 7, 23 y 25. b) PhIC. c) Forófita de Erythrocladia subintegra y Erythrotrichia carnea. d) SC.

Ch. mairei G. Mazoyer

a) 3, 4, 5, 7, 18, 21 y 27. b) HP. c) Epífita de Zostera marina, Z. noltii y Cymodocea nodosa. d) M.

Halopithys incurvus (Huds.) Batters $=H$. pinastroides (S.G. Gmelin) Kütz.

a) MD. b) PhIT. c) Forófita de Acrosorium venulosum, Heterosiphonia crispella, Crouania attenuata y Ceramium flaccidum. d) Abt.

Herposiphonia secunda (C. Agardh) Ambronn

a) MD. b) PhIC. c) Epífita de Cystoseira crinita, Jania rubens, Gelidium pusillum, Acetabularia acetabulum, Cladostephus, Dilophus spiralis, Cystoseira compressa y Sargassum vulgare. d) $\mathrm{P}$.

H. secunda fma. tenella (C. Agardh) Wynne

a) $3,4,5,7,15,18,20,23,24,28$ у 34 . b) PhIC. c) Epífita de Zostera marina, Gelidium pusillum, Cystoseira compressa, Cymodocea nodosa y Cladostephus spongiosus. d) P.

* Laurencia microcladia Kütz.

a) 2, 3, 9, 10, 11 y 20. b) PhIT. c) Forófita de Erythrotrichia sp. pl. y Audouinella parvula.d) At.

L. obtusa (Huds.) Lamour.

a) $2,3,4,5,9,15,18,19,20,21,23,24,25 \mathrm{y}$ 28. b) PhI. c) Forófita de Blidingia marginata, Enteromorpha multiramosa, Ceramium diaphanum, C. tenerrimum y diversas Bangiales. d) C.

* L. paniculata (C. Agardh) J. Agardh

a) 2, 3, 9, 14 y 18. b) Plataformas litorales 
dominadas por Laurencia pinnatifida. d) SC.

* L. papillosa (C. Agardh) Grev.

a) $2,3,9,10,11,12,14,16,17,18,22,24,27$ y 34. b) FM. c) Forófita de Heterosiphonia crispella, Antithamnion cruciatum, Centroceras clavulatum y Audouinella daviesii. d) P.

L. pinnatifida (Gmel.) Lamour.

a) MD. b) PhIB. d) SC.

* L. undulata Yamada
a) $3,9,18,22$ y 27 . b)
b) EM. d) IP.

* Lophosiphonia cristata Falkenb.

a) $2,3,9,10,15,19$ y 20. b) Litoral e infralitoral superior. c) Epífita de Padina pavonica. d) P.

\section{* L. reptabunda (Suhr ex Kütz.) Kylin} $=$ L. obscura (C. Agardh) Falkenb.

a) 1, 3, 11, 22, 23 y 27. b) FM. c) Epífita de Pterocladia capillacea, Gelidium sesquipedale, Gigartina pistillata, Corallina elongata, Cystoseira mediterranea y Cymodocea nodosa. d) SC.

* Osmundaria volubilis (L.) Norris = Vidalia volubilis (L.) J. Agardh a) 3. b) AS. d) At.

Polysiphonia atlantica Kapraun \& Norris $=$ P. macrocarpa Harv. in Mackay

a) 32. b) Ab.

* P. brodiaei (Dillwyn) Spreng.

a) 13 y 16. b) PhIB. c) Epífita de Cystoseira spinosa y Pterosiphonia complanata. d) APtf.

* P. breviarticulata (C. Agardh) Zanardini

a) 2, 3, 12 y 13. b) PhIC. c) Epífita de Dictyota dichotoma var. implexa, Cladostephus spongiosus y Sargassum vulgare. d) M.

* P. deludens Falkenb.

a) 16, 17 y 22. b) PhIB. c) Epífita de Cystoseira tamariscifolia. d) M.

* P. denudata (Dillwyn) Grev. ex Harv. in Hook. $=P$. variegata $(\mathrm{C}$. Agardh $)$ Zanardini

a) MD. b) PhI. c) Epífita de Coralináceas ramificadas e incrustantes, Cystoseira sp.pl. y Gelidiáceas. d) SC.

\section{* P. dichotoma Kütz.}

a) $2,3,15,18,19,20,21,24,25$ y 28. b) PhIC. c) Epífita de Dictyota dichotoma, Zonaria tournefortii, Cystoseira sauvageauana, Codium elongatum, C. bursa y Dictyopteris polypodioides. d) $\mathrm{M}$.

P. elongata (Huds.) Spreng.

a) MD. b) CC. c) Epífita en Cystoseira compressa, Halopteris filicina, Gelidium latifolium, Coralináceas ramificadas y Grateloupia filicina. d) Abt.

P. fucoides (Huds.) Grev. $=P$. violacea $($ Roth) Sprengel

a) $3,5,6,8,18,25$, y 26. b) RMM1. d) Ab.

P. furcellata (C. Agardh) Harv.

a) 34. c) Epífita de Jania rubens y Valonia utricularis. d) APtf.

* P. opaca (C. Agardh) Moris et De Not.

a) MD. b) RM. c) Epífita de Cystoseira tamariscifolia, Hypnea musciformis, Corallina elongata, C. officinalis, Colpomenia sinuosa, Jania rubens y Callithamnion granulatum. d) Ab.

* P. polyspora (C. Agardh) J. Agardh

a) 3, 10, 11 y 20. b) FM. c) Epífita de Ceramium nodulosum, C. ciliatum var. robustum y Jania rubens. d) $\mathrm{M}$.

* P. sertularioides (Grateloup) J. Agardh d) $\mathrm{CB}$

a) 9, 14 y 27. c) Epífita de Gelidium pusillum.

* P. tripinnata J. Agardh d) $\mathrm{M}$.

a) 2, 13 y 21. c) Epífita de Corallina elongata.

* Pterosiphonia complanata (Clemente) Falkenb. a) $16,17,20,22,23,27$ y 28. c) Epífita de Cystoseira tamariscifolia. d) Ab.

* P. parasitica (Huds.) Falkenb.

a) 19, 27 y 28. b) SI. c) Epífita de Gigartina pistillata, Corallina elongata y Sargassum vulgare. d) Ab. 
* P. pennata (C. Agardh) Falkenb.

a) MD. b) SSB. d) SC.

* Rytiphloea tinctoria (Clemente) C. Agardh a) 3. b) PhIT. d) IAt.

\section{PORPHYRIDIALES}

* Chroodactylon ornatum (C. Agardh) Drew et Ross

$=$ Ch. ramossum (Thwaites in Harv.) Hangenot; Asterocystis ornata (C. Agardh) Hamel

a) 1, 16, 22 y 28. c) Epífita de Boergeseniella thuyoides, Jania longifurca, Cladophora coelothrix y Corallina elongata. d) C.

Stylonema alsidii (Zanardini) Drew

a) MD. b) ISR. d) C.

S. cornu-cervi Reinsch

a) $3,4,5,9,11,15,18,19,22,24,27,28$ у 32 .

b) SI. c) Epífita de Peyssonnelia sp.pl., Schottera nicaensis, Gymnogongrus crenulatus, Pterocladia capillacea y Cladophora prolifera. d) APo.

\section{BANGIALES}

Bangia atropurpurea (Roth) C. Agardh

a) $2,5,7,8,13,25$ y 26. b) RMS. d) C.

Porphyra leucosticta Thur. in Le Jolis

a) 34. b) RMS. d) SC.

P. umbilicalis (L.) J. Agardh

a) $8,9,25$ y 34 . b) RMS.

\section{ERYTHROPELTIDALES}

Erythropeltis subintegra (Rosenvinge) Kornmann et Sahling

= Erythrocladia subintegra Rosenvinge; $E$. irregularis Rosenvinge

a) 2, 4, 8, 9, 12, 18, 25 y 26. b) ETN. c) Epífita de Cladophora sp. pl., Bryopsis corymbosa y Derbesia tenuissima. d) C.

Erythrotrichia carnea (Dillwyn) J. Agardh

$=E$. bertholdii Batters; E. investiens (Zanardini) Born.; E. obscura Berthold; E. reflexa (H. M. et P. L. Crouan) Thur. ex De Toni
a) MD. b) ETN. d) C.

* E. rosea P. Dang.

a) 9,13 y 15 . b) HSPP. c) Epífita de Cladophora lehmanniana, Halopteris filicina y Amphiroa beauvoisii. d) M.

\section{FUCOPHYCEAE}

\section{ECTOCARPALES}

* Acinetospora crinita (Carmich. ex Harv.) Sauv. $=$ A. vidovichii (Menegh.) Sauv.

a) 2, 3, 9, 10. b) PhIC. c) Epífita de Cystoseira crinita, C. spinosa y Sargassum vulgare. d) M.

* Ectocarpus fasciculatus Harv., var. pycnocarpus (Rosenvinge) Cardinal

a) $16,17,18,22,23$ y 28. c) Epífita de Phyllariopsis brevipes, Cymodocea nodosa, Halopteris scoparia y Cladostephus spongiosus. d) Ab.

E. siliculosus (Dillwyn) Lyngb. $=$ E. confervoides Le Jolis

a) $1,3,5,8,9,12,19,20,25$ y 26. b) PhIP. c) Epífita de Gracilaria verrucosa, Scytosiphon simplicissimus, Cystoseira crinita, C. compressa, Sargassum vulgare, Cymodocea nodosa, Zostera noltii, Halopteris filicina, Amphiroa beauvoisii, Corallina elongata y Codium grex dichotomus. d) $\mathrm{SC}$.

* E. siliculosus var. penicillatus C. Agardh

a) 1, 3, 8, 19, 23 y 26. b) PhIP, c) Epífita de Codium tomentosum, C. fragile, Laurencia pinnatifida, Colpomenia sinuosa y Ceramium ciliatum var. robustum. d) Ab.

* Feldmannia battersiides (Ercegovic) Cormaci et Furnari

a) 3 y 4. b) PhI. c) Epífita de Cystoseira crinita y Cymodocea nodosa. d) M.

F. globifera (Kütz.) Hamel

a) 3, 4, 5, 6, 7, 8, 15, 23 y 24. b) PhI. c) Epífita de Acetabularia acetabulum, Ulváceas, Cladoforáceas, Dictyotáceas y Fucales. d) SC. 
F. irregularis (Kütz.) Hamel

a) MD. b) Litoral e infralitoral. C) Epífita de Cystoseira crinita, C. compressa, C. tamariscifolia, C. elegans, Sargassum sp.pl., Halopteris filicina, Zostera marina, Cymodocea nodosa yCaulerpa prolifera. d) C.

H. mitchelliae (Harv.) Silva

$=$ G. mitchelliae (Harv.) Hamel

a) MD. b) PhIP. c) Epífita de Cystoseira crinita, C. compressa, Amphiroa beauvoisii, Scytosiphon simplicissimus, Dictyota dichotoma, Codium tomentosum y C. vermilara. d) C.

* H. ovata (Kjellm.) Silva $=G$. intermedia (Rosenvinge) Lund

a) $9,18,23$ y 26 . b) Litoral e infralitoral superior. c) Epífita de Ulva olivascens, Codium elongatum, Jania rubens y Gelidium pusillum. d) M.

* Mesospora macrocarpa (J. Feldm.) Hartog $=M$. mediterranea $\mathrm{J}$. Feldm.

a) MD. b) RMS. d) M.

* Nemoderma tingitanum Schousb. ex Born. a) 2, 3, 10 y 20. b) FM. d) At.

* Ralfsia verrucosa (Areschoug) J. Agardh a) $2,9,10,15,18,20$ y 24 . b) RMI. d) APtf.

\section{DICTYOSIPHONALES}

Asperoccocus bullosus Lamour.

$=$ A. turneri $(\mathrm{Sm}$.) Hook.

a) 4,5 y 7 . b) SRh. d) SC.

\section{SCYTOSIPHONALES}

* Colpomenia peregrina (Sauv.) Hamel

a) $10,11,22$ y 25 . b) PhIP. d) CBA.

C. sinuosa (G. Mertens ex Roth) Derbès et Solier

a) MD. b) PhIP. c) Forófita de Coralináceas incrustantes, Ceramiáceas, Bangiales y Ectocarpáceas. d) C.

* Petalonia fascia (O.F. Müll.) Kuntze a) 8,26 y 27. b) PhIP. d) C.
Scytosiphon simplicissimus (Clemente) Cremades $=S$. lomentaria (Lyngb.) Link

a) $4,5,8,15,20,25,27$ y 28 . b) RMS y PhIP.

c) Forófita de Ectocarpáceas. d) C.

\section{CUTLERIALES}

* Cutleria adspersa (Roth) De Not.
a) $1,12,14$ y 24 . b) PhIP. d) CB.

* C. multifida (Sm.) Grev.
a) 13 y 24. b) PhIP. d) SC.

* Aglaozonia parvula (Grev.) Zanardini

a) $1,8,9,13,18,19,20,24,25$ y 26. b) PhIP.

c) Epífita de Mesophyllum lichenoides y Pseudolithophyllum expansum. d) SC. e) Esporófito de Cutleria multifida (Sm.) Grev.

\section{SPHACELARIALES}

Discosporangium mesarthrocarpum (Menegh.) Hauck

a) 34. b) SCI. e) Citada para la zona por Conde (1984); no ha vuelto a ser recolectada.

* Sphacelaria brachygonia Mont.

a) 2, 3 y 14. b) Litoral e infralitoral superior. c) Epífita de Cystoseira mediterranea y Gelidium latifolium. d) SC.

S. cirrosa (Roth) C. Agardh $=S$. hystrix Suhr ex Reinke

a) MD. b) PhI. c) Epífita de Dictyota dichotoma var. intricata, Cystoseira tamariscifolia, C. spinosa, Sargassum vulgare y Cladostephus spongiosus. d) SC.

\section{S. fusca (Huds.) S.F. Gray}

a) MD. b) Litoral e infralitoral superior. c) Epífita de Padina pavonica, Cystoseira sp.pl., Corallina elongata, Jania rubens y Dictyotáceas. d) SC.

\section{* S. plumula Zanardini}

a) 27 y 28. b) CCT. c) Epífita de Gigartina pistillata. d) Ab.

S. rigidula Kütz.

= S. furcigera Kütz. 
a) MD. b) SSBc. c) Epífita de Peyssonelia squamaria, Pterosiphonia pennata, Cladophora prolifera, Pterocladia capillacea, Schottera nicaensis, Gracilaria verrucosa, Peyssonnelia bornettii y $P$. squamaria. d) C.

S. tribuloides Menegh.

a) 3, 5, 8, 9, 14, 22, 24 y 26. b) PhIT. c) Epífita sobre moluscos y Codium tomentosum. d) C.

Halopteris filicina (Grateloup) Kütz.

a) MD. b) PhI y en cubetas litorales. c) Forófita de varias Ceramiáceas. d) APtf.

H. scoparia (L.) Sauv.

= Stypocaulon scoparium (L.) Kütz.

a) MD. b) PhIC. d) SC.

Cladostephus spongiosus (Huds.) C. Agardh f. verticillatus (Lightf.) P. v. R.

a) $\mathrm{MD}$. b) (PhIC). c) Forófita de Cladoforáceas, Audouinella sp.pl., Ceramiáceas, Cianofíceas y Diatomeas. d) CBA.

\section{DICTYOTALES}

Dictyopteris polypodioides (D.C.) Lamour. $=D$. membranacea (Stackh.) Batters

a) MD. b) SI. d) C.

Dictyota dichotoma (Huds.) Lamour.

a) MD. b) PhIC. c) Forófita de Ectocarpáceas, pequeñas Coralináceas incrustantes, Ceramiáceas y Bangiales. d) C.

D. dichotoma var. intricata (C. Agardh) Grev. $=D$. dichotoma var. implexa (Desf.) J. Agardh

a) MD. b) PhIC. c) Forófita de Fosliella farinosa, Coralináceas incrustantes, Erythrotrichia sp.pl. y Stylonema sp. pl. d) SC.

* D. linearis (C. Agardh) Grev.

$=D$. divaricata Lamour.

a) $2,3,9,10,11,12,13,14,16$ y 17. b) SC. d) P.

* Dilophus fasciola (Roth) M. Howe

a) $3,10,14,15,18,20,21,23,25$ у 28 . b)

PhIC. c) Epífita deSargassum vulgare y Cystoseira sp.pl. d) IAo.
D. spiralis (Mont.) Hamel

$=D$. ligulatus (Kütz.) J. Feldm.

a) MD. b) PhIC. d) Aḅt.

Padina pavonica (L.) Lamour.

a) MD. b) PhIC. c) Forófita de múltiples Ceramiáceas y Coralináceas incrustantes. d) P.

* Spatoglossum solieri (Chauv. ex Mont.) Kütz.

a) 27. b) SCI. d) APo.

Taonia atomaria (Woodw.) J. Agardh

a) 4, 5, 15, 24 y 27. b) PhIC. c) Forófita de Titanoderma pustullatum y Valonia utricularis. d) Abt.

Zonaria tournefortii (Lamour.) Mont.

a) $3,4,5,15,18,19,20,24,25$ y 27 . b) $\mathrm{SCI}$

c) Forófita de varias Ceramiáceas. d) At.

\section{LAMINARIALES}

Phyllariopsis brevipes (C. Agardh) Henry et South $=$ Phyllaria reniformis (Lamour.) Rost.

a) $9,15,16,18,22,27,28$ y 33. b) SRh. c)

Forófita de Ectocarpus fasciculatus, Acrosorium venulosum e Hypoglossum hypoglossoides. d) Ab.

Saccorhiza polyschides (Lightf.) Batters

$=S$. bulbosa (Huds.) Pylaie

a) $9,17,29$ y 33. b) Infralitoral. c) Forófita de Ectocarpáceas. d) Ab.

\section{FUCALES}

Cystoseira amentacea Bory var. stricta Mont. $=$ C. stricta (Mont.) Sauv.

a) 2, 9, 10, 13, 16, 17 y 22. b) PhIB. c) Forófita de Pterosiphonia complanata, Boergeseniella thuyoides, B. fruticulosa y Jania longifurca. d) M.

C. brachycarpa J. Agardh emend Giaccone var. balearica (Sauv.) Giaccone $=$ C. caespitosa Sauv.

a) 3, 18, 22 y 27. b) PhIC. c) Forófita de Centroceras clavulatum y Chaetomorpha linum. d) $\mathrm{M}$.

C. compressa (Esper) Gerloff et Nizamuddin $=C$. fimbriata (Desf.) Bory 
a) MD. b) PhIC. c) Forófita deTitanoderma pustullatum, Fosliella farinosa, Ceramium sp. pl., Enteromorpha sp.pl., Cladophora sp.pl. , Cianofíceas y Diatomeas. d) Ab.

C. crinita (Desf.) Bory

a) $3,4,5,7,15$ y 20 . b) PhIC y cubetas litorales. d) $\mathrm{M}$.

C. elegans Sauv.

a) $1,2,15,18,19,20,21,22,23,27$ y 28 . b) PhIC. c) Forófita de Jania rubens y J. corniculata. d) M.

C. humilis Kütz.

a) $21,22,23,27$ y 28. b) PhI, cubetas de poca profundidad. d) Ab.

C. mauritanica Sauv.

a) $5,9,12,22,23,27$ y 28 . b) PhI. d) Ab. e) Incluida $C$. gibraltarica (Sauv.) Dangeard.

C. mediterranea Sauv.

a) 2, 3 y 34 . b) PhIB. c) Forófita de Polysiphonia sp.pl., Titanoderma sp.pl., Jania sp.pl. y algunas Ceramiáceas. d) $\mathrm{M}$.

C. sauvageauana Hamel

$=C$. selaginoides $\mathrm{Valiante}$

a) 3,15, 19, 20, 27 y 28. b) Lugares calmos y arenosos. d) M.

C. schiffneri Hamel

$=C$. ercegovicii Giaccone

a) $3,9,15,18$ y 20 . b) PhIC y cubetas litorales. c) $\mathrm{M}$.

C. spinosa Sauv.

a) $2,10,15,18,20$ у 34 . b) SCIT. d) M.

C. tamariscifolia (Huds.) Papenfuss

$=C$. ericoides $(\mathrm{L}$.) C. Agardh

a) MD. b) PhIB. c) Forófita de Jania longifurca y Boergeseniella thuyoides. d) Ab.

C. usneoides (L.) Roberts

$=C$. concatenata $\mathrm{C}$. Agardh

a) 17,26 y 28 . b) Infra y circalitoral. d) Ab.

C. zosteroides (Turner) J. Agardh

a) 17 y 22. b) SRh. d) M.
Sargassum acinarium (L.) C. Agardh $=S$. linifolium (Turn.) C. Agardh

a) 4. b) PhIC. c) Forófita de Bangiales, Fosliella farinosa. d) At.

S. desfontaneisii (Turner) C. Agardh

a) 15,19 y 20. b) PhIT. d) At.

S. hornschuchii C. Agardh

a) 3, 20, 27 y 30. b) PhIC. d) M.

S. vulgare C. Agardh

a) MD. b) PhIT. c) Forófita de Alsidium corallinum, Spyridia filamentosa, Hypnea musciformis, Fosliella farinosa, Herposiphonia secunda, Ceramium sp.pl., Feldmannia sp.pl., Cianofíceas y Diatomeas. d) P.

Fucus spiralis L.

a) $18,20,22,28$ y 33. b) Fotófilo en comunidades de Lithophyllum lichenoides y Rissoella verruculosa. d) Ab.

F. vesiculosus $L$.

a) 4. d) Ab.

\section{CHLOROPHYCEAE}

\section{ULOTHRICHALES}

Ulothrix flacca (Dillwyn) Thur. in Le Jolis

a) 32. b) RMS. c) Epífita de Jania corniculata.

d) APtf.

* U. subflaccida Wille

a) $10,13,14$ y 22. b) PhI. c) Epífita de Corallina elongata, Gastroclonium clavatum e Hypnea musciformis. d) APo.

Blidingia marginata (J. Agardh) P. Dang.

a) 5, 17, 10, 20 y 26. b) PhI y cubetas litorales.

c) Epífita de Cystoseira crinita y Sargassum vulgare. d) Ab.

B. minima (Näg. ex Kütz.) Kylin

a) 34. b) PhIP.

Enteromorpha clathrata (Roth) Grev.

a) 5, 8, 9, 18, 20 y 26. b) PhIC. c) Epífita de 
Gracilaria verrucosa e Hypnea musciformis. d) C.

E. flexuosa (Wulfen ex Roth) J. Agardh

a) MD. b) PhIP. c) Epífita de Amphiroa beauvoisii, Scytosiphon simplicissimus, Halopteris filicina., Laurencia pinnatifida, L. obtusa, Cystoseira compressa, C. crinita, Sargassum vulgare y Chondria dasyphylla. d) C.

E. flexuosa subsp. paradoxa (C. Agardh) Bliding a) 34. c) Epífita de Sargassum sp. d) Ab.

E.intestinalis (L.) Link in Nees

a) MD. b) PhIP. d) C. e) Incluye E compressa

(L.) Grev.

* E. kylinii Bliding.
a) 9,15 y 20 . b) PhI. d) Ab.

E. linza (L.) J. Agardh

d) $\mathrm{C}$.

a) $3,7,15,18,19,20,21,24,27$ y 28 . b) PhIC.

\section{E. multiramosa Bliding}

a) MD. b) $\mathrm{PhIC}$. c) Epífita de Spyridia filamentosa, Hypnea musciformis, Taonia atomaria y diversas Fucales. d) M.

E. muscoides (Clemente) Cremades

= E. crinita (Roth) J. Agardh; E. ramulosa (Sm.) Carmichael

a) 2, 16, 22 y 34. b) $\mathrm{PhI}$ ). c) Epífita de Cystoseira tamariscifolia y Pterocladia capillacea. d) SC.

E. prolifera (O.F. Müll.) J. Agardh

a) MD. b) ETN. c) Epífita de las praderas de fanerógamas, Cystoseira sp.pl. y Sargassum vulgare. d) Ab. e) Incluye E. torta (G. Mertens) Reinbold.

E. simplex (Vinogradova) Koeman et Hoek a) 30 y 34. b) ETN.

Ulva cribosa Schousb. ex Born.

a) 33 .

U. curvata (Kütz.) De Toni

a) 34. d) Ab.
U. olivascens P. Dang.

a) 5, 6, 8, 9, 18 y 26. b) PhI. c) Forófita de Amphiroa beauvoisii, Jania rubens y Erythrotrichia carnea. d) Ab.

U. rigida C. Agardh

a) MD. b) PhIP. c) Forófita de Ceramium diaphanum y Jania rubens. d) C.

\section{* Entocladia viridis Reinke}

$=$ Phaeophyla viridis (Reinke) Burrows

a) $2,3,9,11,12,14,15,17,18,19,20,22,23$, 25, 27 y 28. b) SI. c) Epífita de Gracilaria foliifera, Nitophyllum charybdaeum, Laurencia obtusa, Acrosorium venulosum, Dasyopsis plana, Pneophyllum lejolisii y Zonaria tournefortii. d) C.

* Phaeophyla dendroides (P. L .et H. M. Crouan) Batters

a) 3, 20, 27 y 28. b) SI. c) Endófita de Grateloupia filicina, Gracilaria armata, Pneophyllum lejolisii y Gigartina pistillata. d) Ab.

* Pringsheimiella scutata (Reinke) Marchew.

a) 3, 9, 10, 19, 20 y 34. b) PhI. c) Epífita deCymodocea nodosa, Posidonia oceanica y Cladophora prolifera. d) Abt.

Pseudopringsheimia confluens (Rosenvinge) Wille

= Ulvella confluens Rosenvinge

a) 34 .

\section{CLADOPHORALES}

Chaetomorpha gracilis Kütz.

a) 3, 12, 18, 27 y 34. b) RM c) Epífita de Cystoseira tamariscifolia y Cladophora coelothrix. d) Abt.

C. linum (O.F. Müll.) Kütz.

a) MD. b) RM. d) C. e) Incluye a $C$. aerea (Dillwyn) Kütz.

Cladophora albida (Nees) Kütz.

a) 34. d) CB.

C. coelothrix Kütz.

$=C$. repens $(\mathrm{J}$. Agardh) Harv. 
a) MD. b) SSBc. c) Forófita de Erythrotrichia carnea, Ceramium cingulatum, Audouinella sp.pl., Stylonema sp.pl., Antithamnion cruciatum y Platythamnion plumula. d) IAo.

C. dalmatica Kütz.

a) MD. b) RMM2. d) Abt.

C. echinus (Biasoletto) Kütz.

a) $4,5,6,7,12,15,18,20,21,24,25$ y 28 . b) PhIC. c) Epífita de Cladostephus spongiosus, Halopteris filicina y Cystoseira crinita. d) IP.

C. globulina (Kütz.) Kütz.

a) 4, 5, 6 у 7. b) En fondos fangosos con Caulerpa prolifera. d) M.

* C. hutchinsiae (Dillw.) Kütz.

a) $9,10,12,14,18,20,21,24$ y 27 . b) SCI y en pequeñas cubetas litorales. d) APo.

C. Iehmanniana (Lindenb.). Kütz.

$=$ C. ramulosa Menegh; $C$. utriculosa Kütz.

a) 1, 3, 4, 5, 9, 14, 17, 18, 19, 20, 22, 24 у 27. b) SCI y litoral inferior. c) Epífita de Pterocladia capillacea, Gelidium latifolium, Schottera nicaensis, Cystoseira tamariscifolia y Bryopsis plumosa. d) Ab.

C. pellucida (Huds.) Kütz.

a) $4,5,14,18,22,32$ y 34 . b) SSB. d) Ab.

C. prolifera (Roth) Kütz.

a) $2,3,4,5,9,15,19,20,21,27$ y 34 . b) AS.

c) Frecuentemente epífita de Coralináceas incrustantes. d) Abt. e) Asociada casi constante con Schmitziella endophloea, dándole un aspecto rojizo a su talo.

\section{C. rupestris (L.) Kütz.}

$=$ C. ramosissima (Draparnaud ex Kütz.) Kütz.

a) 3, 11, 12, 20, 23, 27 y 34. b) SI. c) Epífita de Peyssonnelia squamaria y Pterocladia capillacea. d) Ao.

* C. sericea (Huds.) Kütz.

a) $10,14,16,17,18,22,23$ y 27 . b) ETN; fotófila del medio e infralitoral superior. c) Epífita de Cystoseira tamariscifolia, Laurencia pinnatifida y Mytilus sp. d) CB.
C. vadorum (Areschoug) Kütz.

a) $4,5,6,7,9,12$ y 18 . b) $\mathrm{PhIT}$, en pequeñas cubetas litorales calcáreas. d) Abt.

C. vagabunda (L.) Hoek

a) $3,4,5,10,18,19,23,27$ y 28 . b) RM y en pequeñas cubetas. c) Epífita de Padina pavonica, Colpomenia sinuosa, Spyridia filamentosa y Gelidium pusillum. d) Abt.

Rhizoclonium tortuosum (Dillw.) Kütz. $=R$. riparium (Roth) Harv.

a) MD. b) ETN. d) C.

\section{SIPHONOCLADALES}

Valonia macrophysa Kütz.

a) $3,11,18$ y 34 . b) SCI. d) P.

V. utricularis (Roth) C. Agardh

a) MD. b) SSR. d) P.

Ventricaria ventricosa (J. Agardh) Olsen et J.A. West

= Valonia ventricosa J. Agardh

a) 34. d) P.

\section{DASYCLADALES}

Acetabularia acetabulum (L.) Silva $=A$. mediterranea Lamour.

a) $1,2,3,4,5,7,9,10,11,13,15$ y 34 . b)

PhIC. d) IAt.

* A. calyculus Lamour.

a) 5. d) P.

* Polyphysa parvula (Solms) Schnetter et Bula-Meyer Solms = Acetabularia moebii Solms; A. parvula

a) 3 y 9. b) SSBc. d) IP.

* Dasycladus vermicularis (Scopoli) Krasser $=D$. clavaeformis (Roth) C. Agardh

a) 3. b) PhIT, en fondos arenosos o arenosofangosos. d) At.

CODIALES (BRYOPSIDALES)

* Bryopsidella neglecta (Berthold) Rietema 
= Derbesia neglecta Berthold

a) 2, 3, 11 y 27. b) Litoral. d) M.

* Bryopsis corymbosa J. Agardh

a) 2, 3, 9, 17 y 21. b) SI. c) Epífita de Peyssonnelia squamaria. d) M.

* B. duplex De Not.

$=$ B. balbisiana Lamour. sensu J. Feldm.

a) 13, 14, 16, 22, 23 y 28. b) PhIB. c) Epífita de Pterocladia capillacea, Corallina elongata, Gelidium latifolium y Patella safiana. d) Ab.

* B. feldmannii Gallardo et Furnari

$=$ B. cupressoides Kütz. sensu J. Feldm.

a) 9, 10, 11 y 15. b) SI. c) Epífita de Amphiroa cryptarthrodia. d) M.

* B. hypnoides Lamour.

$=B$. monoica Berthold ex Funk

a) $9,12,13,14,16,18,22,24$ y 27 . b) SCI. c) Epífita de Pterocladia capillacea, Cladophora prolifera, Mesophyllum lichenoides, Amphiroa rigida y Sargassum vulgare. d) C.

* B. muscosa Lamour.

a) $3,9,14,20,22$ y 34 . b) EM. d) M.

B. plumosa (Huds.) C. Agardh

a) MD. b) PhIP. d) IPo.

Derbesia tenuissima (De Not.) H. M. et P. L. Crouan

a) MD. b) ETN, litoral e infralitoral. c) Epífita de Cystoseira compressa, C. crinita, Sargassum vulgare, Enteromorpha compressa, Corallina elongata y Amphiroa beauvoisii. d) Ab.

* Halicystis parvula F. Schmitz in Murray

a) $3,14,20$ y 27 . b) SIC. c) Epífita de Coralináceas incrustantes. d) Ab. e) Gametófito de Derbesia tenuissima.

Pedobesia lamourouxii (J. Agardh) J. Feldm., Loreau, Codomier et Couté

= Derbesia lamourouxii (J. Agardh) Solier

a) 4, 5 y 8. b) HSPP. c) Epífita de Gelidium pusillum. d) At.

*P. solieri Feldm. ex Abelard et Knoep-Pég. a) 1, 3 y 9. b) Litoral e infralitoral superior.

Codium adhaerens (Cabrera) C. Agardh

a) $3,9,10,12,17,18,20,22,23,27,28$ у 33 .

b) SC. d) IAo.

* C. bursa (L.) C. Agardh

a) $3,8,9,11,13,15,18,20$ y 22 . b) PhIC. c) Forófita de pequeñas Coralináceas incrustantes, Jania rubens, Centroceras clavulatum y otras Ceramiáceas. d) Abt.

* C. decorticatum (Woodw. ) M. Howe $=$ C. elongatum (Turn.) C. Agardh

a) $2,3,9,12,15,18,19,20,21,23,24,27 \mathrm{y}$

28. b) HSPP, hasta $23 \mathrm{~m}$ de profundidad. d) Ao.

C. effusum (Rafinesque) Delle Chiaje $=C$. difforme $\mathrm{Kütz}$.

a) $3,4,5,9,11,13,14,15,22,23$ y 28 . b) SCI.

d) IAo.

* C. fragile (Suringar) Hariot

a) 8. b) PhIG. c) Forófita de Audouinella codii, A. saviana y Ceramium sp.pl. d) APtf.

C. tomentosum Stackh.

a) $2,3,8,15,18,23,26$ y 33. b) PhIP. c) Forófita de Ceramiáceas y Audouinella codii. d) APo.

C. vermilara (Olivi) Delle Chiaje

a) $8,13,14,15,18,20,22,24,26$ y 34 . b) $\mathrm{SCI}$.

c) Forófita de Ceramiáceas (Spermothamnion flabellatum), Acrochaetiáceas y Dasyáceas. d) Atf.

* Halimeda tuna (J. Ellis et Sol.) Lamour.

a) 34. b) AS. c) Epífita en los rizomas de Posidonia oceanica. d) P.

Flabellia petiolata (Turra) Nizamuddin = Udotea petiolata (Turra) Boerg.

a) 3 y 34. b) AS. c) Epífita en los rizomas de Posidonia oceanica . d) At.

Caulerpa prolifera (Forsskaal) Lamour.

a) 4, 5, 6 y 7. b) PhIM. c) Forófita de Polysiphonia elongata, Erythrotrichia carnea y Hincksia mitchelliae. d) At. 


\section{LILIOPSIDA}

\section{NAJADALES}

Posidonia oceanica (L.) Delile

a) 34. b) PhIM. c) Forófita, a nivel de hoja, de Fosliella farinosa, Titanoderma pustullatum, Ceramium tenerrimum y Ectocarpus siliculosus y, a nivel de rizomas, de Flabellia petiolata, Halimeda tuna, Peyssonnelia rubra y Jania rubens. d) M. e) Lozano Cabo (1953) la cita en la Mar Chica donde cremos que ha desaparecido en la actualidad.

\section{Cymodocea nodosa (Ucria) Asch.}

a) $3,4,5,7,18,19,20,21,27$ y 28 ; arrojada en la mayoría de las estaciones. b) Fotófilo infralitoral de sustratos móviles, PhIM. c) Forófita de Chondria capillaris, C. mairei, Ceramiáceas y Ectocarpáceas. d) Ab.

\section{Zostera marina L.}

a) $2,4,5,6,7,9,13,15,18,20$ y 28. b) PhIM. c) Forófita de Ectocarpáceas, Ceramiáceas, Chondria sp.pl., Fosliella farinosa y Coralináceas incrustantes. d) C.

Z. noltii Hornem.
a) $3,4,5,6$ y 7 .
b) PhIM.
d) Ab.

\section{TAXA EXCLUDENDA}

Caulacanthus ustulatus (G. Mertens) Kütz.

Probablemente, las cita de este tax on hay que referirlas a Feldmannophycus rayssiae (J. Feldm. et G. Feldm.) Augier et Boudouresque, ambas especies son morfológicamente muy parecidas (Augier \& Boudouresque, 1971).

Cystoseira baccata (S.G. Gmel.) Silva

Según González García y Conde (1993), esta especie no se encuentra en la zona estudiada. Las referencias sobre ella se deben a incorrectas identificaciones.

\section{Ulva lactuca $L$.}

Segun Bliding (1968) y Rizzi \& Giaccone (1974) no es una especie mediterránea; las citas sobre ella corresponden a Ulva rigida o $U$. olivascens

\section{TAXA INQUIRENDA}

* Hincksia dalmatica (Ercegovic) Cormaci et Furnari Furnari

$=$ Giffordia dalmatica (Ercegovic) Cormaci et

Probable forma atípica de $H$. mitchelliae, de la que se diferencia por la forma e inserción de los zoidocistos.

\section{Laminaria rodriguezii Born.}

a) 9. b) SRh. d) M. e) Citada, procedente de dragados, por Sauvageau (1920) y Bellón (1925). No ha vuelto a ser recolectada.

Phyllariopsis purpurascens (C. Agardh) Henry et South

= Phyllaria purpurascens $($ C. Agardh) Rost.

a) 9. b) Ab. e) Citada, procedente de dragados, por Sauvageau (1920) y Bellón (1925). No ha vuelto a ser recolectada.

Cystoseira foeniculacea (L.) Grev.

a) 9 y 33. b) Ab. e) Citada por Werner (1962); su presencia no ha sido confirmada en nuestras recolecciones.

C. platyclada Sauv.

a) 9 y 12. b) M. e) Su presencia en la zona ha sido puesta en duda por Ribera et al. (1992).

\section{BIBLIOGRAFÍA}

AUGIER, H. \& C.F. BOUDOURESQUE -1971Découverte des cystocarpes Feldmannophycus rayssiae (J. Feldm. et G. Feldm.) nov. gen. (Rhodophycées), Gigartinales. Soc. Phycol. de France, Bull., 16: 25-30.

ARDRÉ, F. -1969/70- Contribution à l'étude des algues marines du Portugal. I. - La Flore. Port. Act. Biol. (B), 10(1-4): 137-532.

BELLÓN, L. -1925- Nota sobre una Sacchoriza bulbosa (Hudson) La Pyl. de Melilla (Mediterráneo occidental). Nuova Notarisia, 217-221.

BLIDING, G. -1968- A critical survey of European taxa in Ulvales. II.- Ulva, Ulvaria, Monostroma, 
Kormannia. Bot. Notar., 121: 535-629.

BOUDOURESQUE, C.F. -1984- Groupes écologiques d'algues marines et phytocenoses benthiques en Méditerranée Nord-occidentale: une revue. Giorn. Bot. Ital., 118(2): 7-42.

BRESSAN, G. -1974- Rodoficee calcaree dei mari italiani. Boll. Soc. Adriat. Scienc. Trieste, 59(2): $1-132$.

BUÉN, O. DE -1912- Notas sobre la biología y aprovechamiento de Mar Chica. Anuario de Pesca y Estadística de la Marina Mercante y de la Pesca del año 1911.

BUÉN, O. DE -1913- Notes sur les fonds et sur la pêche dans la côte méditerranéenne du Riff. Atti V Congrs. Internaz di Pesca. Roma.

BURROWS, E.M. -1991-Seaweeds of the British Isles. 2. Chlorophyta. Natural History Museum. London. 238 pp.

CONDE, F. -1984- Contribución al conocimiento de la flora bentónica del Mar de Alborán. Islas Chafarinas. Acta Bot. Malacitana, 9: 41-46.

CONDE, F. -1991- Catálogo y comentarios sobre el «complejo Audouinella» (Acrochaetiaceae, Rhodophyceae) en el Mediterráneo. Cryptogamie Algol., 12(3): 163-170.

DEBRAY, F. -1897- Catalogue des algues $d u$ Maroc, d'Algerie et de Tunisie. A. Jourdan Edit. Alger., 78 pp.

ERCEGOVIC, A. -1956- Famille des Champiacées dans l'Adriatique Moyenne. Acta Adriatica, 8(2): 1-16.

FUNK, G. -1955- Beiträge zur Kenntnis der Meeresalgen von Neapel Zugleich mikrophotographischer atlas. Pubbl. Staz. Zool. Napoli, 25 supl., (1-10): 215-230.

GALlARDO, T., A. GÓMEZ GARRETA, M.A. RIBERA, M. ÁLVAREZ \& F. CONDE - 1985A preliminary checklist of Iberian benthic marine algae. Real Jardín Botánico. Madrid. 83 pp.

GALlARDO, T., A. GÓMEZ GARRETA, M.A. RIBERA, M. CORMACI, G. FURNARI, G. GIACCONE \& C.F. BOUDOURESQUE - 1993 Checklist of Mediterranean Seaweeds II. Chlorophyceae Wille, s.l. Bot. Mar., 36: 399421.

GAYRAL, P. - 1966- Les algues des côtes françaises. Doin. Paris. 632 pp.

GIACCONE, G., P. COLONNA, C. GRAZIANO, A. MANNINO, E. TORNATORE, M.
CORMACI G. FURNARI \& B. SCAMMACCA -1985-Revisione della flora marina di Sicilia e isole minori. Boll. Acc. Gioenia Sci. Nat., 18(326): 537-781.

GONZÁLEZ GARCÍA, J.A., y F. CONDE -1991Estudio florístico, fenológico, autoecológico y fitogeográfico del macrofitobentos de la Mar Chica (Sebcha Buareg de Nador, Mediteráneo marroquí). Acta Bot. Malacitana, 16(1): 63-80.

GONZÁLEZ GARCÍA, J.A., y F. CONDE -1992Sargassum desfontainesii (Turner) C. Agardh (Fucales, Fucophyceae), primera cita para el Mediterráneo. Acta Bot. Malacitana, 17: 250251.

GONZÁLEZ GARCÍA, J.A., y F. CONDE -1993Estudio biogeográfico de las Fucales y Laminariales atlánticas en el litoral mediterráneo de Marruecos. Acta Bot. Malacitana, 18: 39-44.

HAMEL, G. \& P. LEMOINE - 1952- Corallinacées de France et d'Afrique du Nord. Arch. Mus. Nat. Histor., 7e Sér. I: 17-131, 83 fig. 23 pl.

HARIOT, M.P. -1909- Sur une collection d'algues recueillies à Maroc par $\mathrm{M}$. Buchet. Bull. Mus. Hist. Nat., 3: 128-130.

HARIOT, M.P. -1911- Sur une collection d'algues recueillies à Mauritania par $\mathrm{M}$. Chudeau. Bull. Soc. Bot. Fr., 58: 438-445.

LEMOINE, P. -1926- Corallinacées du Maroc II. Bull. Soc. Sc. Nat. Maroc, 6(1-6): 106-108.

LOZANO CABO, F. -1953- Notas sobre una prospección pesquera en la Mar Chica. Bol. Inst. Esp. Ocean., 64: 3-37.

MAGGS, C.A. \& M.H. HOMMERSAND -1993Seaweeds of the British Isles. Vol. 1. Rhodophyta. Part 3A Ceramiales. The Natural History Museum. London. $444 \mathrm{pp}$.

NAVARRO, M.J. y T. GALLARDO -1989- Aportación al conocimiento de la flora bentónica de las costas mediterráneas africanas occidentales. Bot. Complutensis, 15: 203-214.

PERRET-BOUDOURESQUE, M., \& H SERIDI 1989-Inventaire des algues marines benthiques d'Algerie. GIS Posidonie Publ., Marseille, Fr., 1: 1-117.

RIBERA, M.A., A. GÓMEZ GARRETA, T. GALLARDO, M. CORMACI, G. FURNARI \& G. GIACCONE - 1992- Checklist of Mediterranean Seaweeds. I. Fucophyceae (Warming, 1884). Bot. Mar., 35: 109-130. 
RIZZI, L. \& G. GIACCONE -1974- Le Ulvale e la vegetazione nitrofila del Mediterraneo. Pubbl. Univ. Trieste Ist. Bot., supl. 5(2): 3-62 + 19 fig.

SAUVAGEAU,, C. -1913- Sur les Fucacées du étroit de Gibraltar. Comp. Rend. Hebd. Séances Acad. Sci., 157: 1539-1540.

SAUVAGEAU, C. -1920- A propos des Cystoseira de Banyuls et de Guétary. Bull. Stat. Biol. Arcachon, 17: 3-51.

SILVA, P.C. \& H.W. JOHANSEN -1986- A reappraisal of the order Corallinales (Rhodophyceae). Br. Phycol. J., 21: 245-254.

SOTO, J. y F. CONDE -1989- Catálogo florístico de las algas bentónicas marinas del litoral de Almería. Bot. Complutensis, 15: 61-83.

SOTO, J. y F. CONDE - 1990- Algunas consideraciones sobre la flora algal marina de Murcia (sureste de España). Bentos, 6: 15-21.

VERLAQUE, M. \& H. SERIDI -1991- Antithamnion algeriensis nov.sp. (Ceramiaceae, Rhodophyta) from Argelia (Mediterranean Sea). Bot. Mar., 34: 153-160.
WERNER, R.G. -1962- Essai d'une étude de la répartition des Cryptogames marines et maritimes du Maroc. Bull. Soc. Sci. Nat. \& Phys. Maroc, 42: 1-33.

WOELKERLING, W.J. \& L.M. IRVINE -1982The genus Schmitziella Born. et Batters (Rhodophyta): Corallinaceae or Acrochaetiaceae?. Br. Phycol. J., 17: 275-295.

Aceptado para su publicación en Junio de 1994

Dirección de los autores. J.A. González García: Escuela Universitaria de Formación del Profesorado. Melilla. F. Conde Poyales: Dpto. Biología Vegetal. Fac. Ciencias. Universidad de Malaga. Ap. 59. 29080-Málaga. 